Portland State University

PDXScholar

\title{
Fourier Transform of the Multicenter Product of 1s Hydrogenic Orbitals and Coulomb or Yukawa Potentials and the Analytically Reduced Form for Subsequent Integrals that Include Plane Waves
}

Jack C. Straton

Portland State University, straton@pdx.edu

Follow this and additional works at: https://pdxscholar.library.pdx.edu/phy_fac

Part of the Atomic, Molecular and Optical Physics Commons

Let us know how access to this document benefits you.

\section{Citation Details}

Straton, Jack C. "Fourier transform of the multicenter product of 1 s hydrogenic orbitals and Coulomb or Yukawa potentials and the analytically reduced form for subsequent integrals that include plane waves." Physical Review A 39.10 (1989): 5062. DOI: http://dx.doi.org/10.1103/PhysRevA.39.5062

This Article is brought to you for free and open access. It has been accepted for inclusion in Physics Faculty Publications and Presentations by an authorized administrator of PDXScholar. Please contact us if we can make this document more accessible: pdxscholar@pdx.edu. 


\title{
Fourier transform of the multicenter product of $1 s$ hydrogenic orbitals and Coulomb or Yukawa potentials and the analytically reduced form for subsequent integrals that include plane waves
}

\author{
Jack C. Straton* \\ Laboratory for Astronomy and Solar Physics, Goddard Space Flight Center, \\ National Aeronautics and Space Administration, \\ Greenbelt, Maryland 20771 \\ (Received 28 January 1988)
}

\begin{abstract}
The Fourier transform of the multicenter product of $N 1 s$ hydrogenic orbitals and $M$ Coulomb or Yukawa potentials is given as a $(M+N-1)$-dimensional Feynman integral with external momenta and shifted coordinates appearing as quadratic forms $P$ and $S$ in $(P / S)^{v} K_{v}(P S)$, where $K_{v}$ is a modified Bessel function of the second kind. This is accomplished through the introduction of an integral transformation, in addition to the standard Feynman transformation for the denominators of the momentum representation of the terms in the product, which moves the resulting denominator into an exponential. This allows the angular dependence of the denominator to be combined with the angular dependence in the plane waves. All angular dependence is then removed by invoking an orthogonal transformation that does not need to be explicitly calculated. The extension to excited states is outlined. The class of integrals over the shifted coordinates, containing plane waves in addition to this product of orbitals and potentials, is given in analytically reduced form, with the external momenta appearing as $S^{-\gamma}$.
\end{abstract}

\section{INTRODUCTION}

A large class of problems in atomic and molecular physics depends on the evaluation of integrals composed of a product of hydrogenic orbitals, Coulomb or Yukawa potentials, and (possibly) plane waves. One of the central techniques in reducing such matrix elements to tractable numerical form has been to Fourier transform parts of the integrand and then to combine the angular dependence using Feynman parametrization. ${ }^{1}$ The tedium and the possibilities for error of this approach grow with the increasing complexity of the problem, so it would be helpful to have a unified prescription for carrying out both the Fourier and Feynman transformations as a systematic intermediate step for subsequent integration over arbitrary functions. The present paper not only gives the Fourier transform for a general multicenter product of $1 s$ hydrogenic orbitals, Coulomb or Yukawa potentials, including the necessary Feynman parametrization, but also succeeds in carrying out the subsequent integration, once and for all, for the entire class of integrals in which these arbitrary functions are plane waves.

The general formula for the Fourier transform of a hydrogenic orbital of an arbitrary state was first found by Podolsky and Pauling. ${ }^{2}$ Recently, Straton ${ }^{3}$ has found an analytic form for the Fourier transform of a one-center product of $N$ orbitals (of an arbitrary state), required for calculating matrix elements involving bound-state projection operators such as orthogonalization corrections in charge transfer. ${ }^{4}$ An analytic form for three-electron integrals, a special case of the Fourier transform with the momentum variable equal to zero, has been found by Fromm and Hill. ${ }^{5}$ Fourier transforms of pairs of orbitals centered at different points have been given in various forms containing one-dimensional integrals. ${ }^{6-10}$ or in infinite series. ${ }^{11}$ Of particular note is the technique of representing hydrogenic orbitals by a finite sum of $B$ functions, ${ }^{9,12-14}$ which have exceedingly simple Fourier transforms. ${ }^{15}$

In this paper the integral transform method is extended to the calculation of the Fourier transform of a multicenter product of $N$ hydrogenic orbitals of the ground state and $M$ Coulomb or Yukawa potentials,

$$
\begin{array}{r}
I_{1 s, \ldots, 1 s}^{\lambda_{1}, \ldots, \lambda_{N} ; \eta_{1}, \ldots, \eta_{M}}\left(\mathbf{K}, \mathbf{R}_{1}, \ldots, \mathbf{R}_{M}, \mathbf{R}_{M+1}, \ldots, \mathbf{R}_{M+N}\right) \\
=\frac{1}{(2 \pi)^{3 / 2}} \int d^{3} r e^{-i \mathbf{K} \cdot \mathbf{r}} V_{\eta_{1}}\left(\mathbf{r}-\mathbf{R}_{1}\right) \cdots V_{\eta_{M}}\left(\mathbf{r}-\mathbf{R}_{M}\right) \\
\quad \times u_{1 s}^{\lambda_{1}}\left(\mathbf{r}-\mathbf{R}_{M+1}\right) \cdots u_{1 s}^{\lambda_{N}}\left(\mathbf{r}-\mathbf{R}_{M+N}\right),
\end{array}
$$

and the modifications required to extend this to products including excited states are discussed. Note that the symmetrical normalization is used so that the inverse transform is

$I \ldots(\mathbf{r}, \ldots)=\frac{1}{(2 \pi)^{3 / 2}} \int d^{3} K e^{i \mathbf{K} \cdot \mathbf{r}} \boldsymbol{I} \ldots(\mathbf{K}, \ldots)$.

The final number of Feynman integrals depends on the number of products in (1), so if any of the $\mathbf{R}_{j}$ are identical, the product of these terms should be rewritten as an orbital or potential with $\lambda$ or $\eta$ being the sum of the constituent $\lambda_{i}$ and $\eta_{j}$. This transform is a useful intermediate step in the analytical reduction of the general class of atomic and molecular integrals ${ }^{16}$ in which the atoms are centered at different points so that the $\mathbf{R}_{i}$ are linear combinations of the variables of integration $\mathbf{x}_{j}$, 


$$
\begin{aligned}
M_{\sigma_{1} \sigma_{2} \cdots \sigma_{N}}^{\lambda_{1} \cdots \lambda_{N} ; \eta_{1} \cdots \eta_{M}}\left(\mathbf{K}, \mathbf{p}_{1}, \ldots, \mathbf{p}_{m}\right)=\int d^{3} x_{1} \cdots d^{3} x_{m} e^{-i\left(\mathbf{p}_{1} \cdot \mathbf{x}_{1}+\cdots+\mathbf{p}_{m} \cdot \mathbf{x}_{m}\right)} I_{\sigma_{1} \sigma_{2} \cdots \lambda_{N}}^{\lambda_{1} \cdots \lambda_{N} ; \eta_{1} \cdots \eta_{M}} \\
\\
\quad \times\left(\mathbf{K}, \mathbf{R}_{1}, \ldots, \mathbf{R}_{M}, \mathbf{R}_{M+1}, \ldots, \mathbf{R}_{M+N}\right) T\left(\mathbf{K}, \mathbf{x}_{1}, \ldots, \mathbf{x}_{m}, \mathbf{p}_{1}, \ldots, \mathbf{p}_{m}\right),
\end{aligned}
$$

where $K$ and the p's may be zero, $T$ is an arbitrary function, and $m \leq N+M$ [otherwise some of the $\mathbf{R}_{j}$ must be identical and (1) could be rewritten with a smaller value for $M+N]$.

In Sec. II an orthogonal transformation method for integrating a product of Feynman propagators ${ }^{17}$ is generalized to allow integrals containing angular dependence in both plane waves and in a product of denominators that are quadratic in the integration variables. This transformation is invoked as a systematic method equivalent to completing the square simultaneously in all momentum integrals. But since the final form, after the threedimensional momentum integrals are evaluated (like their four-dimensional counterparts in the original application), depends only on the determinant of the transformed quadratic form, which is equal to the determinant of the original quadratic form, the transformation does not need to be explicitly calculated.

This result is used in Sec. III to calculate (1), and the extension to products containing excited states is outlined in Sec. IV. Finally, Sec. V includes a discussion of how to treat the angular dependence of any subsequent integrals over the vectors $\mathbf{R}_{j}$. The integrals in (2) are evaluated ex- plicitly for the common class of problems in which $T \equiv 1$ and $\sigma_{j}=1 s$ by again invoking an orthogonal transformation that does not need to be actually computed.

\section{GENERAL CLASS OF INTEGRALS}

The Fourier transform of the product (1) may be evaluated by convolution methods. One first introduces the Fourier transforms of the individual terms (in atomic units)

$$
u_{1 s}^{\lambda}(\mathbf{x})=\left[\frac{\lambda}{\pi}\right]^{5 / 2} \int d^{3} k \frac{e^{i \mathbf{k} \cdot \mathbf{x}}}{\left(\lambda^{2}+k^{2}\right)^{2}}
$$

where

$$
\lambda=\frac{Z}{a_{0}}
$$

in which $a_{0}$ is the Bohr radius, and ${ }^{18}$

$$
V_{\eta}(\mathbf{x})=\frac{e^{-\eta x}}{x}=\frac{1}{2 \pi^{2}} \int d^{3} k \frac{e^{i \mathbf{k} \cdot \mathbf{x}}}{\left(\eta^{2}+k^{2}\right)}, \quad \eta \geq 0
$$

Then

$$
\begin{aligned}
I= & \left(\frac{\lambda_{1}}{\pi} \frac{\lambda_{2}}{\pi} \cdots \frac{\lambda_{N}}{\pi}\right]^{5 / 2} \frac{(2 \pi)^{3 / 2}}{\left(2 \pi^{2}\right)^{M}} e^{-i \mathbf{K} \cdot \mathbf{R}_{M+N}} \\
& \times \int d^{3} k_{1} d^{3} k_{2} \cdots d^{3} k_{M} d^{3} k_{M+1} d^{3} k_{M+2} \cdots d^{3} k_{M+N-1} \frac{e^{-i\left[\mathbf{k}_{1} \cdot\left(\mathbf{R}_{1}-\mathbf{R}_{M+N}\right)+\mathbf{k}_{2} \cdot\left(\mathbf{R}_{2}-\mathbf{R}_{M+N}\right)+\cdots+\mathbf{k}_{M} \cdot\left(\mathbf{R}_{M}-\mathbf{R}_{M+N}\right)\right]}}{\left(\eta_{1}^{2}+k_{1}^{2}\right)\left(\eta_{2}^{2}+k_{2}^{2}\right) \cdots\left(\eta_{M}^{2}+k_{M}^{2}\right)} \\
& \times \frac{e^{-i\left[\mathbf{k}_{M+1} \cdot\left(\mathbf{R}_{M+1}-\mathbf{R}_{M+N}\right)+\mathbf{k}_{M+2} \cdot\left(\mathbf{R}_{M+2}-\mathbf{R}_{M+N}\right)+\cdots+\mathbf{k}_{M+N-1} \cdot\left(\mathbf{R}_{M+N-1}-\mathbf{R}_{M+N}\right)\right]}}{\left(\lambda_{1}^{2}+k_{M+1}^{2}\right)^{2} \cdots\left(\lambda_{M+N-1}^{2}+k_{M+N-1}^{2}\right)^{2}\left[\lambda_{M+N}^{2}+\left(\mathbf{k}_{1}+\cdots+\mathbf{k}_{M+N-1}-\mathbf{K}\right)^{2}\right]^{2}}
\end{aligned}
$$

This is a special case of a more general class of integrals

$$
J_{L}\left(\mathbf{B}_{i}, \mathbf{p}_{i}, s_{i}\right)=\int d^{3} k_{1} d^{3} k_{2} \cdots d^{3} k_{L} \frac{e^{-i\left(\mathbf{k}_{1} \cdot \mathbf{B}_{1}+\mathbf{k}_{2} \cdot \mathbf{B}_{2}+\cdots+\mathbf{k}_{L} \cdot \mathbf{B}_{L}\right)}}{\left(s_{1}+q_{1}^{2}\right)^{1+m_{1}}\left(s_{2}+q_{2}^{2}\right)^{1+m_{2}} \cdots\left(s_{n}+q_{n}^{2}\right)^{1+m_{n}}},
$$

where $\mathbf{q}_{i}$ is a linear combination of external momenta $\mathbf{p}_{j}$ and at least one internal momentum vector $\mathbf{k}_{j}$.

To integrate over the $k_{j}$, first introduce the standard integral transform for the denominators ${ }^{19}$ generalized to allow arbitrary powers of the denominators,

$$
\frac{1}{D_{1}^{1+m_{1}} D_{2}^{1+m_{2}} \cdots D_{n}^{1+m_{n}}}=\frac{\left(n+\sum_{i} m_{i}-1\right) !}{\left(m_{1}\right) !\left(m_{2}\right) ! \cdots\left(m_{n}\right) !} \int_{0}^{1} d \alpha_{1} \int_{0}^{1} d \alpha_{2} \cdots \int_{0}^{1} d \alpha_{n} \alpha_{1}^{m_{1}} \alpha_{2}^{m_{2}} \cdots \alpha_{n}^{m_{n}} \frac{\delta\left[1-\sum_{i=1}^{n} \alpha_{i}\right]}{\left(\sum_{i=1}^{n} \alpha_{i} D_{i}\right)^{n+\sum_{i} m_{i}} .}
$$

This allows the angular dependence in the denominators to be combined into a single quadratic form. This technique has been used extensively in atomic and molecular problems in the simpler case of $n=2,{ }^{20}$ and in particular for the Fourier transforms of the $B$ functions, in which other exponential type functions may be expanded. ${ }^{6}$

Chisholm ${ }^{17}$ analyzed integrals over four-dimensional analogues of these denominators, arising from Feynman propagators, and developed a systematic approach, useful in the present three-dimensional case, that will be extended to in- 
clude the angular dependence of the plane waves in the numerator of (7). This is done by introducing the additional transformation ${ }^{21}$

$$
(v-1) ! D^{-v}=\int_{0}^{\infty} d \rho \rho^{v-1} e^{-\rho D}
$$

which moves the angular dependence of the denominator in (8) into an exponential so that the angular dependence of the plane waves in (7) may be included.

At this stage one could complete the square for each $\mathbf{k}_{i}$ in $D$, change variables in $D, \mathbf{k}_{i} \cdot \mathbf{B}_{i}$, and $\int d^{3} k_{i}$, and then integrate. But this iterative process is tedious and error prone. An additional complication is that in succeeding integrals the coefficient of $k_{j}^{2}$ is no longer simply $\alpha_{j}$ but contains subtractions that may be large enough to make the coefficient negative for some values of $\alpha$. In such a case the integral (17) is no longer well defined.

Instead, multiply and divide the $B$ 's by $\rho$ so that all $k$ dependence may be written in terms of a single quadratic form $Q$ in

$$
\begin{aligned}
J_{L}\left(B_{i}, p_{i}, s_{i}\right)=\frac{1}{\left(m_{1}\right) !\left(m_{2}\right) ! \cdots\left(m_{n}\right) !} \int_{0}^{1} d \alpha_{1} \int_{0}^{1} d \alpha_{2} \cdots \int_{0}^{1} d \alpha_{n} \alpha_{1}^{m_{1}} \alpha_{2}^{m_{2}} \cdots \alpha_{n}^{m_{n}} \delta\left[1-\sum_{i=1}^{n} \alpha_{i}\right] \\
\\
\times \int_{0}^{\infty} d \rho \rho^{n+\sum m_{i}-1} \int d^{3} k_{1} d^{3} k_{2} \cdots d^{3} k_{L} e^{-\rho Q} .
\end{aligned}
$$

The quadratic form may be written in a compact form

$$
Q=\underline{V}^{T} \underline{W} \underline{V},
$$

where

$$
\underline{V}^{T}=\left(\mathbf{k}_{1}, \mathbf{k}_{2}, \ldots, \mathbf{k}_{L}, 1\right),
$$

and

$$
\underline{W}=\left[\begin{array}{ccccc}
a_{11} & a_{12} & \cdots & a_{1 L} & \mathbf{b}_{1} \\
a_{21} & a_{22} & \cdots & a_{2 L} & \mathbf{b}_{2} \\
\vdots & \vdots & & \vdots & \vdots \\
a_{L 1} & a_{L 2} & \cdots & a_{L L} & \mathbf{b}_{L} \\
\mathbf{b}_{1} & \mathbf{b}_{2} & \cdots & \mathbf{b}_{L} & C
\end{array}\right] .
$$

In this compact notation the $a_{i j}$ are just linear combinations of the Feynman parameters $\alpha_{j^{\prime}}$,

$$
\mathbf{b}_{j} \equiv \mathbf{v}_{j}+i \frac{\mathbf{B}_{j}}{2 \rho}
$$

where $\mathbf{v}_{j}$ is a linear combination of the $\mathbf{p}_{i}$, and $C$ contains those parts of the denominator on the right-hand side of (8) that are independent of the variables $\mathbf{k}_{i}$.

A simple example is

$J_{1}\left(\mathbf{B} ; \mathbf{p}_{1}, \mathbf{p}_{2} ; s_{1}, s_{2}\right)=\int d^{3} k \frac{e^{-i \mathbf{k} \cdot \mathbf{B}}}{\left(s_{1}+q_{1}^{2}\right)\left(s_{2}+q_{2}^{2}\right)}$,

where

$$
\mathbf{q}_{j}=\mathbf{k}-\mathbf{p}_{j} .
$$

Then $\underline{W}$ is a $2 \times 2$ matrix with

$$
\begin{aligned}
& \mathbf{b}_{1}=-\alpha_{1} \mathbf{p}_{1}-\alpha_{2} \mathbf{p}_{2}+i \frac{\mathbf{B}}{2 \rho}, \\
& a_{11}=\alpha_{1}+\alpha_{2} \equiv \Lambda
\end{aligned}
$$

and

$$
C=\alpha_{1}\left(s_{1}+p_{1}^{2}\right)+\alpha_{2}\left(s_{2}+p_{2}^{2}\right) .
$$

Now if one can find an orthogonal transformation that reduces $\underline{Q}$ to diagonal form

$$
Q^{\prime}=a_{1}^{\prime} k_{1}^{\prime 2}+a_{2}^{\prime} k_{2}^{\prime 2}+\cdots+a_{L}^{\prime} k_{L}^{\prime 2}+c^{\prime},
$$

then, as shown by Chisholm, ${ }^{17}$ the $a_{j}^{\prime}$ are positive. Then after a simple translation in $\left\{\mathbf{k}_{1}, \mathbf{k}_{2}, \ldots, \mathbf{k}_{N}\right\}$ space (with Jacobian $=1$ ), the $k^{\prime}$ integrals may be done using

$$
\int d^{3} k^{\prime} e^{-\rho a^{\prime} k^{\prime 2}}=4 \pi \int_{0}^{\infty} d k^{\prime} k^{\prime 2} e^{-\rho a^{\prime} k^{\prime 2}}=\left(\frac{\pi}{\rho a^{\prime}}\right)^{3 / 2} \text {. }
$$

Then $L$ integrations yield

$$
\int d^{3} k_{1}^{\prime} \cdots d^{3} k_{L}^{\prime} e^{-\rho\left(a_{1}^{\prime} k_{1}^{\prime 2}+\cdots+a_{L}^{\prime} k_{L}^{\prime 2}\right)}=\left(\frac{\pi^{L}}{\rho^{L} \Lambda^{\prime}}\right)^{3 / 2},
$$

where

$$
\Lambda^{\prime}=a_{1}^{\prime} a_{2}^{\prime} \cdots a_{L}^{\prime} .
$$

But the orthogonal transformation leading to (16) leaves the determinants

$$
\Omega=\operatorname{det} \underline{W}
$$

and

$$
\Lambda=\left|\begin{array}{cccc}
a_{11} & a_{12} & \cdots & a_{1 L} \\
a_{21} & a_{22} & \cdots & a_{2 L} \\
\vdots & \vdots & & \vdots \\
a_{L 1} & a_{L 2} & \cdots & a_{L L}
\end{array}\right|
$$

invariant, ${ }^{17}$ so

$$
\Lambda^{\prime}=\Lambda
$$

and

$$
\Omega=a_{1}^{\prime} a_{2}^{\prime} \cdots a_{L}^{\prime} c^{\prime} .
$$


It is seen in the present case, as with the four-dimensional case studied by Chisholm, ${ }^{17}$ that one does not need to know the individual $a_{j}^{\prime}$ of (16), only the product (19) that is completely determined by the known determinant (21). The remaining quantity in (16) is also given by the invariant matrices,

$$
c^{\prime}=\Omega / \Lambda \text {. }
$$

Thus, the transformation that diagonalizes (13) never has to be explicitly calculated.

Before doing the final integral,

$$
\boldsymbol{P}=\int_{0}^{\infty} d \rho \rho^{n+\sum_{i} m_{i}-3 L / 2-1} e^{-\rho c^{\prime}},
$$

the $\rho$ dependence of $c^{\prime}$ must be made explicit. The only $\rho$-dependent quantities are in $\Omega$, in the single term $i\left(\mathbf{B}_{j} / 2 \rho\right)$ of each $\mathbf{b}_{j}$. Expanding $\Omega$ by minors gives

$$
\begin{aligned}
\Omega & =\operatorname{det} \underline{W} \\
& =C \Lambda+\sum_{i=1}^{L} \sum_{j=1}^{L} \mathbf{b}_{i} \cdot \mathbf{b}_{j}(-1)^{i+j+1} \Lambda_{i j} \\
& =\chi+\frac{G}{4 \rho^{2}}+i \frac{F}{2 \rho},
\end{aligned}
$$

where, from (14),

$$
\begin{aligned}
& \chi=C \Lambda-E, \\
& G=\sum_{i=1}^{L} \sum_{j=1}^{L} \mathbf{B}_{i} \cdot \mathbf{B}_{j}(-1)^{i+j} \Lambda_{i j}, \\
& E=\sum_{i=1}^{L} \sum_{j=1}^{L} \mathbf{v}_{i} \cdot \mathbf{v}_{j}(-1)^{i+j} \Lambda_{i j}, \\
& F=2 \sum_{i=1}^{L} \sum_{j=1}^{L} \mathbf{B}_{i} \cdot \mathbf{v}_{j}(-1)^{i+j+1} \Lambda_{i j},
\end{aligned}
$$

and where the minor $\Lambda_{i j}$ is $\Lambda$ with the $i$ th row and $j$ th column deleted. For the example given in $\left(7^{\prime}\right)-(15)$, for which one defines $\Lambda_{11} \equiv 1$ since $L=1$,

$$
\begin{aligned}
& G=B^{2}, \\
& E=\left(\alpha_{1} \mathbf{p}_{1}+\alpha_{2} \mathbf{p}_{2}\right)^{2},
\end{aligned}
$$

and

$$
F=2\left(\alpha_{1} \mathbf{p}_{1}+\alpha_{2} \mathbf{p}_{2}\right) \cdot \mathbf{B} .
$$

Chisholm's analysis of quadratic forms resulting from integrals over products of Feynman propagators ${ }^{17}$ applies to the present case with a few modifications due to the introduction of the following complex terms in (14): The determinant $\Lambda(21)$ is positive; if the $k$ 's and $p$ 's are not imaginary and if some $s$ is nonzero, so that the denominator in (7) is positive definite (nonzero if not all $\mathbf{k}_{j}$ are zero), then $\chi(27)$ is positive definite; $E$ and $G$ are nonnegative.

The $\rho$ integral may now be done, ${ }^{22}$

$$
\begin{aligned}
P= & e^{-i F / 2 \Lambda} \int_{0}^{\infty} d \rho \rho{ }_{i}^{n+\sum_{i} m_{i}-3 L / 2-1} e^{-\rho \chi / \Lambda-G /(4 \Lambda \rho)} \\
= & 2 e^{-i F / 2 \Lambda}\left(\frac{G}{4(C \Lambda-E)}\right)^{\left(n+\sum_{i} m_{i}-3 L / 2\right) / 2} \\
& \times K_{n+\sum_{i} m_{i}-3 L / 2}\left(\frac{\sqrt{G(C \Lambda-E)}}{\Lambda}\right) .
\end{aligned}
$$

Note that (31) is true only for $G / \Lambda$ and $\chi / \Lambda$ positive. In the present case $\chi / \Lambda$ will be zero only if $\alpha_{i}=0$ for all $i$. But this is prevented by the $\delta$ function in (10). However, if all $\mathbf{B}_{i}$ are zero, $G$ and $F$ will be zero so instead of (31) one has

$$
P=\frac{\Gamma\left(n+\sum_{i} m_{i}-3 L / 2\right)}{\chi^{n+\sum_{i} m_{i}-3 L / 2}},
$$

with the restriction $n+\sum_{i} m_{i}>3 L / 2$, which will hold if the original integral (7) is convergent. But this corresponds to the Fourier transform of a product of onecenter hydrogenic orbitals for which an analytical result exists. $^{3}$

The final result is found by integrating one of the $\alpha$ 's, using the $\delta$ function, in

$$
\begin{aligned}
J_{L}\left(B_{i}, p_{i}, s_{i}\right)= & \frac{2 \pi^{3 L / 2}}{\left(m_{1}\right) !\left(m_{2}\right) ! \cdots\left(m_{n}\right) !} \\
& \times \int_{0}^{1} d \alpha_{1} \int_{0}^{1} d \alpha_{2} \cdots \int_{0}^{1} d \alpha_{n} \alpha_{1}^{m_{1}} \alpha_{2}^{m_{2}} \cdots \alpha_{n}^{m_{n}} \delta\left[1-\sum_{i=1}^{n} \alpha_{i}\right] e^{-i F / 2 \Lambda \frac{1}{\Lambda^{3 / 2}}} \\
& \times\left(\frac{G}{4(C \Lambda-E)}\right)^{\left(n+\sum_{i} m_{i}-3 L / 2\right) / 2} K_{n+\sum_{i} m_{i}-3 L / 2}\left[\frac{\sqrt{G(C \Lambda-E)}}{\Lambda}\right) .
\end{aligned}
$$

For the example $\left(7^{\prime}\right)-(15)$ and $\left(28^{\prime}\right)-\left(30^{\prime}\right)$ one integrates $\alpha_{2}$, using the $\delta$ function, so that $\Lambda=1$. Then defining

$$
\mu^{2}=C-E=\alpha s_{1}+(1-\alpha) s_{2}+\alpha(1-\alpha)\left(\mathbf{p}_{1}-\mathbf{p}_{2}\right)^{2},
$$

one has 


$$
\begin{aligned}
J_{1}\left(\mathbf{B} ; \mathbf{p}_{1}, \mathbf{p}_{2} ; s_{1}, s_{2}\right) & =2 \pi^{3 / 2} \frac{\sqrt{B}}{2} e^{-i \mathbf{B} \cdot \mathbf{p}_{2}} \int_{0}^{1} d \alpha e^{-i \alpha \mathbf{B} \cdot\left(\mathbf{p}_{1}-\mathbf{p}_{2}\right)} \frac{K_{1 / 2}(B \mu)}{\sqrt{\mu}} \\
& =\pi^{2} e^{-i \mathbf{B} \cdot \mathbf{p}_{2}} \int_{0}^{1} d \alpha e^{-i \alpha \mathbf{B} \cdot\left(\mathbf{p}_{1}-\mathbf{p}_{2}\right)} \frac{e^{-B \mu}}{\mu}
\end{aligned}
$$

This is exactly the result obtained through bypassing the $\rho$ integral (7) and completing the square in the denominator of $(6),{ }^{23}$

$$
\begin{aligned}
J_{1}\left(\mathbf{B} ; \mathbf{p}_{1}, \mathbf{p}_{2} ; s_{1}, s_{2}\right) & =\int_{0}^{1} d \alpha \int d^{3} k \frac{e^{-i \mathbf{B} \cdot \mathbf{k}}}{\left\{k^{2}-2\left[\alpha \mathbf{p}_{1}-(1-\alpha) \mathbf{p}_{2}\right] \cdot \mathbf{k}+(1-1)\left[\alpha \mathbf{p}_{1}+(1-\alpha) \mathbf{p}_{2}\right]^{2}+C\right\}^{2}} \\
& =e^{-i \mathbf{B} \cdot \mathbf{p}_{2}} \int_{0}^{1} d \alpha e^{-i \alpha \mathbf{B} \cdot\left(\mathbf{p}_{1}-\mathbf{p}_{2}\right)} \int d^{3} k^{\prime} \frac{e^{-i \mathbf{B} \cdot \mathbf{k}^{\prime}}}{\left(k^{\prime 2}+\mu^{2}\right)^{2}} \\
& =e^{-i \mathbf{B} \cdot \mathbf{p}_{2}} \frac{4 \pi}{B} \int_{0}^{1} d \alpha e^{-i \alpha \mathbf{B} \cdot\left(\mathbf{p}_{1}-\mathbf{p}_{2}\right)} \int_{0}^{\infty} d k^{\prime} \frac{k^{\prime} \sin B k^{\prime}}{\left(k^{\prime 2}+\mu^{2}\right)^{2}} .
\end{aligned}
$$

Note that the latter reproduces Cheshire's technique ${ }^{24}$ except that his $\mu^{2}$ erroneously neglects the momentum cross term. However, since he only uses this integral in the case where one of the momenta is zero, this error does not alter his result.

\section{FOURIER TRANSFORM OF THE PRODUCT}

The Fourier transform of a product of $N 1 s$ hydrogenic orbitals and $M$ Coulomb or Yukawa potentials is simply a special case of $J_{L}$. One has only to substitute into (33)

$L=M+N-1, \quad n=M+N$,

$s_{i}=\eta_{i}^{2}, \quad m_{i}=0, \quad i=1,2, \ldots, M$

$s_{i}=\lambda_{i}^{2}, \quad m_{i}=1, \quad i=M+1, M+2, \ldots, M+N$,

$\mathbf{B}_{i}=\mathbf{R}_{i}-\mathbf{R}_{M+N}, \quad q_{i}^{2}=k_{i}^{2}, \quad i=1,2, \ldots, M+N-1$, and

$$
\begin{aligned}
\mathbf{q}_{M+N}=\mathbf{k}_{1}+\mathbf{k}_{2}+ & \cdots+\mathbf{k}_{M}+\mathbf{k}_{M+1} \\
& +\mathbf{k}_{M+2}+\cdots+\mathbf{k}_{M+N-1}-\mathbf{K},
\end{aligned}
$$

which give

$$
\begin{aligned}
& a_{i j}=\alpha_{M+N}+\delta_{i j} \alpha_{i}, \\
& \mathbf{b}_{j}=-\alpha_{M+N} \mathbf{K}+i \frac{\mathbf{B}_{j}}{2 \rho},
\end{aligned}
$$

and

$$
C_{\mathrm{FT}}=\sum_{i=1}^{M} \alpha_{i} \eta_{i}^{2}+\sum_{i=M+1}^{M+N} \alpha_{i} \lambda_{i}^{2}+\alpha_{M+N} K^{2} .
$$

The determinants $\Lambda$ and $\Omega$ are greatly simplified by subtracting the $(M+N-1)$ st row from the previous $M+N-2$ rows,

$$
\begin{aligned}
& \Lambda_{\mathrm{FT}}=\left|\begin{array}{ccccc}
\alpha_{1} & 0 & \cdots & 0 & -\alpha_{M+N-1} \\
0 & \alpha_{2} & & 0 & -\alpha_{M+N-1} \\
\vdots & & & & \vdots \\
0 & 0 & & \alpha_{M+N-2} & -\alpha_{M+N-1} \\
\alpha_{M+N} & \alpha_{M+N} & \cdots & \alpha_{M+N} & \alpha_{M+N}+\alpha_{M+N-1}
\end{array}\right|=\sum_{i=1}^{N+M}\left(\prod_{j \neq i} \alpha_{j}\right),
\end{aligned}
$$

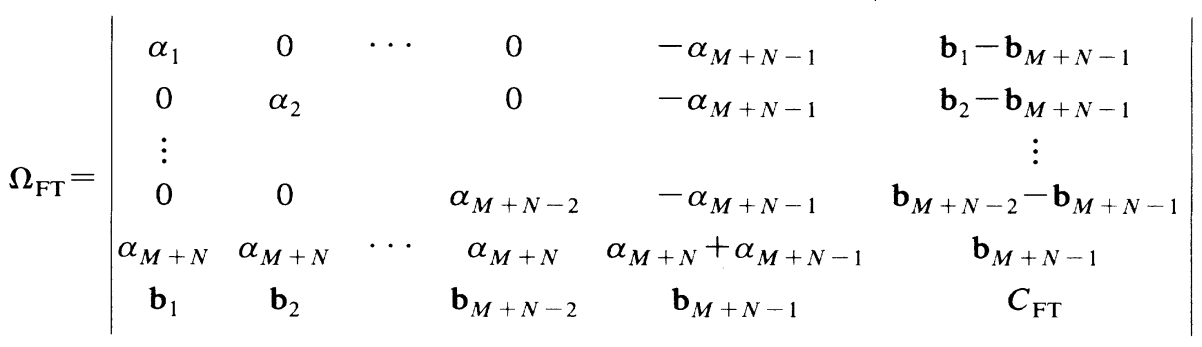

$$
\begin{aligned}
& =C_{\mathrm{FT}} \Lambda_{\mathrm{FT}}-E_{\mathrm{FT}}+i \frac{F_{\mathrm{FT}}}{2 \rho}+\frac{G_{\mathrm{FT}}}{4 \rho^{2}},
\end{aligned}
$$

where

$$
E_{\mathrm{FT}}=\alpha_{M+N}^{2} K^{2} \sum_{i=1}^{M+N-1}\left(\prod_{j \neq i, n+N} \alpha_{j}\right),
$$




$$
\begin{aligned}
& F_{\mathrm{FT}}=2 \alpha_{M+N} \mathbf{K} \cdot \sum_{i=1}^{M+N-1}\left(\prod_{j \neq i, n+N} \alpha_{j}\right) \mathbf{B}_{i}, \\
& G_{\mathrm{FT}}=\sum_{i=1}^{M+N-1}\left[\sum_{j \neq i}\left(\prod_{m \neq i, j} \alpha_{m}\right) B_{i}^{2}-2 \sum_{j=1}^{i-1}\left(\prod_{m \neq i, j} \alpha_{m}\right) \mathbf{B}_{j} \cdot \mathbf{B}_{i}\right] .
\end{aligned}
$$

Substituting (34)-(46) into (33) and multiplying by

$$
\left(\frac{\lambda_{1}}{\pi} \frac{\lambda_{2}}{\pi} \cdots \frac{\lambda_{N}}{\pi}\right)^{5 / 2} \frac{(2 \pi)^{3 / 2}}{\left(2 \pi^{2}\right)^{M}}
$$

gives the final result for (1),

$$
\begin{aligned}
& I_{1 s, \ldots, 1 s}^{\lambda_{1}, \ldots, \lambda_{N} \eta_{1}, \ldots, \eta_{M}}\left(\mathbf{K}, \mathbf{R}_{1}, \ldots, \mathbf{R}_{M}, \mathbf{R}_{M+1}, \ldots, \mathbf{R}_{M+N}\right) \\
& =\frac{\left(\lambda_{1} \lambda_{2} \cdots \lambda_{N}\right)^{5 / 2} 2^{5 / 2-M}}{\pi^{N+M / 2}} \\
& \times \int_{0}^{1} d \alpha_{1} \int_{0}^{1} d \alpha_{2} \cdots \int_{0}^{1} d \alpha_{M+N} \alpha_{M+1} \alpha_{M+2} \cdots \alpha_{M+N} \delta\left(1-\sum_{i=1}^{M+N} \alpha_{i}\right) e^{-i F_{\mathrm{FT}} / 2 \Lambda_{F T}} \frac{1}{\Lambda_{\mathrm{FT}}^{3 / 2}} \\
& \times\left(\frac{G_{\mathrm{FT}}}{4\left(C \Lambda_{\mathrm{FT}}-E_{\mathrm{FT}}\right)}\right)^{(N-M+1) / 4} K_{(N-M+1) / 2}\left(\frac{\sqrt{G_{\mathrm{FT}}\left(C_{\mathrm{FT}} \Lambda_{\mathrm{FT}}-E_{\mathrm{FT}}\right)}}{\Lambda_{\mathrm{FT}}}\right) .
\end{aligned}
$$

\section{EXCITED STATES}

The present procedure may be extended to find the Fourier transform of products of orbitals including excited states in some cases. For $s$ states the coordinate space radial wave function includes a polynomial in the coordinate multiplying an exponential that may be written as a polynomial of derivatives of the exponential with respect to $\lambda_{j}$ [now containing the principal quantum number in the denominator of (4)], which is just a polynomial of derivatives of $1 s$ orbitals. The final expression will then contain a polynomial of derivatives of (33) with respect to the $\lambda_{i}$ in $C$. Alternatively, the momentum representation of $s$ states in general contains a polynomial in $k^{2}$ in the numerator of the equivalent of (3). The equivalent of (33) is found by substituting into the equivalent of (3)

$$
\frac{A+B k^{2}+C k^{4}+\cdots}{\left(\lambda+k^{2}\right)^{n}}=\frac{A}{\left(\lambda+\tau k^{2}\right)^{n}}-B \frac{\partial}{\partial \tau} \frac{1}{(n-1)\left(\lambda+\tau k^{2}\right)^{n-1}}+C \frac{\partial^{2}}{\partial \tau^{2}} \frac{1}{(n-1)(n-2)\left(\lambda+\tau k^{2}\right)^{n-2}}+\cdots,
$$

resulting in $a_{j j} \rightarrow \tau_{j} a_{j j}$ in (13), (21), and (39). Then the final form is given by taking derivatives of (33) or (48) with respect to the $\tau$ 's, for each term in the polynomial, and then setting the $\tau$ 's equal to one.

States with $l>0$ in the product are more difficult to handle. In some problems the product of orbitals includes a projector onto $2 p$-states, ${ }^{4}$

$$
\begin{aligned}
& \sum_{m=-1}^{1} u_{2 l m}^{\lambda_{j}^{*}\left(\mathbf{r}-\mathbf{R}_{j}\right) u_{2 l m}^{\lambda_{j+1}}\left(\mathbf{r}-\mathbf{R}_{j+1}\right)} \\
& \quad=\sum_{m=-1}^{1} \frac{1}{(2 \pi)^{3}}(i)(-i) \frac{4}{3 \pi}\left(\lambda_{j} \lambda_{j+1}\right)^{7 / 2} \int d^{3} k_{j} \int d^{3} k_{j+1} \frac{k_{j} Y_{1 m}^{*}\left(\Omega_{j}\right)}{\left[\left(\lambda_{j} / 2\right)^{2}+k_{j}^{2}\right]^{3}} \frac{k_{j+1} Y_{1 m}\left(\Omega_{j+1}\right)}{\left[\left(\lambda_{j+1} / 2\right)^{2}+k_{j+1}^{2}\right]^{3}} \\
& \quad=\frac{\left(\lambda_{j} \lambda_{j+1}\right)^{7 / 2}}{2^{2} \pi^{5}} \int d^{3} k_{j} \int d^{3} k_{j+1} \frac{\mathbf{k}_{j} \cdot \mathbf{k}_{j+1}}{\left[\left(\lambda_{j} / 2\right)^{2}+k_{j}^{2}\right]^{3}\left[\left(\lambda_{j+1} / 2\right)^{2}+k_{j+1}^{2}\right]^{3}}
\end{aligned}
$$

By rewriting the last term in the denominator of the equivalent of (6),

$$
\begin{aligned}
D_{M+N}= & \lambda_{M+N}^{2}+\left(\mathbf{k}_{1}+\mathbf{k}_{2}+\cdots+\mathbf{k}_{j-1}+\mathbf{k}_{j+2}+\cdots+\mathbf{k}_{M+N-1}-\mathbf{K}\right)^{2} \\
& +\left(\mathbf{k}_{1}+\mathbf{k}_{2}+\cdots+\mathbf{k}_{j-1}+\mathbf{k}_{j+2}+\cdots+\mathbf{k}_{M+N-1}-\mathbf{K}\right) \cdot\left(\mathbf{k}_{j}+\mathbf{k}_{j+1}\right)+k_{j}^{2}+k_{j+1}^{2}+\left.2 \tau \mathbf{k}_{j} \cdot \mathbf{k}_{j+1}\right|_{\tau=1},
\end{aligned}
$$


one may remove the vector product in the numerator by differentiation,

$$
\frac{\mathbf{k}_{j} \cdot \mathbf{k}_{j+1}}{D_{M+N}^{2}}=-\frac{1}{2} \frac{\partial}{\partial \tau} \frac{1}{D_{M+N}} .
$$

Then one may use the present technique by setting $m_{M+N}=0$ in (36), $a_{j, j+1}=\tau \alpha_{M+N}$ in (39), and the $j, j+1$ element of (42) and (43) equal to $(\tau-1)$ rather than 0 , and by taking the derivative of (33) with respect to $\tau$. If the product in the equivalent of (1) contains either more pairs of $p$ states than the value of the principal quantum number of the $N$ th orbital, or if it contains pairs of states with sufficiently high $l$, so that one has higher powers of $\cos \theta_{j, j+1}$ in the equivalent of (50), the transformation (9) may be performed first to allow sufficient powers of the denominator for multiple derivatives of the type (52).

\section{SUBSEQUENT INTEGRALS}

In developing (34)-(48), the solution for the Fourier transform (1), a three-dimensional integral has been replaced by an $(M+N-1)$-dimensional integral. The utility of this intermediate step lies in the ease of evaluating a subsequent $3 m$-dimensional integral over the $x_{j}$ 's (2), where $m \leq N+M$, so that the overall dimensionality is reduced by about $70 \%$. Likewise, (7) may appear in integrals over the "external" momenta $p_{j} .{ }^{25}$

First note that for the simple integral considered in $\left(7^{\prime}\right)$ the final expression (33') allows a change of variables to $\mathbf{p}^{\prime}=\mathbf{p}_{1}-\mathbf{p}_{2}$ in a subsequent integration over momenta. Alternatively, if the subsequent integration is over $d^{3} B$, one may treat the quantity $\mu$ in $\left(33^{\prime}\right)$ as an inverse Bohr radius, rewrite

$$
\frac{e^{-\mu B}}{\mu}=\left(\frac{\pi}{\mu^{5}}\right)^{1 / 2} u_{1 s}^{\mu}(B),
$$

and integrate over $d^{3} B$ using the Fourier transform that is the inverse of (3). If the $B$ integrand contains other hydrogenic orbitals one may use the sequence $(1)-(48)$ recursively.

More generally, for $m>1$, the subsequent integrals over the $B$ 's (linear functions of the $R$ 's that are in turn linear functions of the $x$ 's) may contain other functions of the $x$ 's than just the $G$ and $F$ of (33), such as $T$ in (2). Such cases merit individual attention. But because many problems in atomic physics, particularly scattering problems, ${ }^{16}$ are a simple integral of $J_{L}\left(\mathbf{B}_{i}, \mathbf{p}_{i}, s_{i}\right)$ (7), multiplied by plane waves and integrated over the $x_{j}$, the $I \rightarrow J$ generalization of (2) in the case $T \equiv 1$,

$$
\begin{aligned}
N_{m L}\left(\mathbf{p}_{i}, s_{i}\right)=\int & d^{3} x_{1} \cdots d^{3} x_{m} J_{L}\left(\mathbf{B}_{i}, \mathbf{p}_{i}, s_{i}\right) \\
& \times e^{-i\left(\mathbf{p}_{1} \cdot \mathbf{x}_{1}+\cdots+\mathbf{p}_{m} \cdot \mathbf{x}_{m}\right)},
\end{aligned}
$$

will now be explicitly done.

It is clear from the form (7) that one could integrate first over the $\mathbf{x}_{i}$ yielding a product of $m \delta$ functions inside the $\mathbf{k}_{j}$ integrals, allowing these integrals to be evaluated before applying the transformations (8) and (9). However since

$$
\mathbf{B}_{i}=\sum_{j=1}^{m} t_{i j} \mathbf{x}_{j}
$$

the $\delta$ functions will be of the form

$$
\delta\left[\mathbf{p}_{j}-\sum_{i=1}^{m} t_{i j} \mathbf{k}_{i}\right] .
$$

As the first integral, $d^{3} k_{1}$, is evaluated, one substitutes

$$
\left[\mathbf{p}_{1}-\sum_{i=2}^{m} t_{i 1} \mathbf{k}_{i}\right] / t_{11}
$$

for $\mathbf{k}_{1}$ in (56) for each $j>1$ and in the denominator of (7), which appears in (54). Next, one substitutes

$$
\frac{\mathbf{p}_{2}-\mathbf{p}_{1} / t_{11}-\sum_{i=3}^{m}\left(t_{i 2}+t_{i 1} / t_{11}\right) \mathbf{k}_{i}}{t_{22}+t_{12} / t_{11}}
$$

for $\mathbf{k}_{2}$ in (56) for each $j>2$ and in the denominator of (54), and so on. This process becomes unwieldly and prone to error as $m$ becomes larger, increases the complexity of the angular parts of the $x$ integrals, and does not decrease the number of products in the denominator (nor, hence, the number of Feynman integrals) except where an $\mathbf{x}_{i}$ appears in only one $\mathbf{B}_{j}$ of (55). But that case yields nothing new since one could do these integrals before the $r$ integral using the Fourier inverses of (3) and (5). Also, even when (55) is nontrivial, if all of the $\mathbf{p}_{i}$ are zero and $m=L$ one may change variables of integration in (54) from $\mathbf{x}_{i}$ to $\mathbf{B}_{i}$ and one may again integrate directly.

Consider the more difficult case in which $(55)$ is a nontrivial set or not all of the $\mathbf{p}_{i}$ are zero. From (45) and (46) it is clear that $F$ is a linear function and $G$ is a quadratic function of the $\mathbf{B}_{i}$ and, hence, of the $\mathbf{x}_{j}$. After integrating over the $\mathbf{k}_{i}$ but before integrating over $\rho$, in order to group all angular dependence of the $x$ 's together, one may append the $\rho\left(i \mathbf{p}_{j} \cdot \mathbf{x}_{j} / \rho\right)$ from the plane waves in (54) to the expanded form of $G$ that appears in the exponential on the first line of (31). Then

$$
\begin{aligned}
\frac{G}{(2 \rho)^{2}}+i \frac{F}{2 \rho} \rightarrow H & =\frac{G}{(2 \rho)^{2}}+i \frac{F}{2 \rho}+2 i \sum_{j=1}^{m} \frac{\mathbf{p}_{j} \cdot \mathbf{x}_{j}}{2 \rho} \\
& =-\underline{X}^{T} \underline{Z} \underline{X},
\end{aligned}
$$

where

$$
\underline{X}^{T}=\left[i \frac{\mathbf{x}_{1}}{2 \rho}, i \frac{\mathbf{x}_{2}}{2 \rho}, \ldots, i \frac{\mathbf{x}_{m}}{2 \rho},-1\right],
$$

and

$$
\underline{Z}=\left[\begin{array}{ccccc}
z_{11} & z_{12} & \cdots & z_{1 m} & \mathbf{h}_{1} \\
z_{21} & z_{22} & & z_{2 m} & \mathbf{h}_{2} \\
\vdots & & & & \vdots \\
z_{m 1} & z_{m 2} & & z_{m m} & \mathbf{h}_{m} \\
\mathbf{h}_{1} & \mathbf{h}_{2} & \cdots & \mathbf{h}_{m} & 0
\end{array}\right] .
$$

As with (13), the elements of (61) are completely known, 
given by (59). The $h$ 's are linear combinations of $K$ and the $p$ 's and the $z_{i j}$ are functions of the $\alpha$ 's [for the Fourier transform, for example, they are linear combinations of the coefficients of the vectors in (45) and (46)].

As with (16), suppose one finds an orthogonal transformation that reduces $H$ to diagonal form,

$$
\begin{aligned}
H^{\prime} & =-i^{2}\left(z_{1}^{\prime} x_{1}^{\prime 2}+z_{2}^{\prime} x_{2}^{\prime 2}+\cdots+z_{m}^{\prime} x_{m}^{\prime 2}\right) / 4 \rho^{2}+g^{\prime} \\
& \equiv \frac{\gamma}{4 \rho^{2}}+g^{\prime},
\end{aligned}
$$

where $g^{\prime}$, the quadratic form in the external momenta $\mathbf{p}_{j}$, and $z_{i}^{\prime}$ are non-negative. ${ }^{26}$ But the postulated transformation leaves the determinants of the quadratic form invariant so

$$
z_{1}^{\prime} z_{2}^{\prime} \cdots z_{m}^{\prime} \equiv \Delta=\left|\begin{array}{cccc}
z_{11} & z_{12} & \cdots & z_{1 m} \\
z_{21} & z_{22} & & z_{2 m} \\
\vdots & & & \vdots \\
z_{m 1} & z_{m 2} & \cdots & z_{m m}
\end{array}\right| .
$$

giving

$$
g^{\prime}=|\underline{Z}| / \Delta \equiv \zeta / \Delta
$$

Then substituting (26), (59), (62), and (64) into (24) gives

$$
\rho c^{\prime}=\rho(\chi+\zeta / \Delta) / \Lambda+\gamma /(4 \Lambda \rho),
$$

yielding (33) with $F \rightarrow 0, C \Lambda-E \rightarrow C \Lambda-E+\zeta / \Delta$, and $G \rightarrow \gamma$. Finally one may integrate (7) over the $x$ 's of $\gamma$, after a translation in $\left\{\mathbf{x}_{1}, \mathbf{x}_{2}, \ldots, \mathbf{x}_{m}\right\}$ space, using ${ }^{27}$

$$
\begin{gathered}
\int d^{3} x\left[\sqrt{z}\left(x^{2}+\beta / z\right)^{1 / 2}\right]^{-v} K_{-v}\left[\mu \sqrt{z}\left(x^{2}+\beta / z\right)^{1 / 2}\right] \\
=4 \pi \int_{0}^{\infty} d x x^{2}\left[\sqrt{z}\left(x^{2}+\beta / z\right)^{1 / 2}\right]^{-v} \\
\quad \times K_{v}\left(\mu \sqrt{z}\left(x^{2}+\beta / z\right)^{1 / 2}\right] \\
=\frac{(2 \pi)^{3 / 2}}{(\mu z)^{3 / 2}\left(\beta^{v-3 / 2}\right)^{1 / 2}} K_{v-3 / 2}(\mu \sqrt{\beta})
\end{gathered}
$$

where

$$
-v=n+\sum_{i} m_{i}-3 L / 2, \mu=\frac{\sqrt{C \Lambda-E+\xi / \Delta}}{\Lambda},
$$

except for the last integral, in which $\beta_{m}=0$, that is given by $^{28}$

$$
\begin{gathered}
\int d^{3} x_{m}^{\prime} z_{m}^{\prime[-v+3(m-1) / 2] / 2} x_{m}^{\prime-v+3(m-1) / 2} K_{v-3(m-1) / 2}\left(\mu x_{m}^{\prime} \sqrt{z_{m}^{\prime}}\right) \\
=4 \pi \int_{0}^{\infty} d x_{m}^{\prime} z_{m}^{\prime[-v+3(m-1) / 2] / 2} x_{m}^{\prime-v+3(m-1) / 2+2} K_{v-3(m-1) / 2}\left(\mu x_{m}^{\prime} \sqrt{\left.z_{m}^{\prime}\right)}\right. \\
=\frac{\pi^{3 / 2} 2^{-v+(3 m+1) / 2} \Gamma(-v+3 m / 2)}{z_{m}^{\prime 3 / 2} \mu^{-v+3(m+1) / 2}}
\end{gathered}
$$

The $m$ integrations yield the factor

$$
\frac{1}{\left(z_{1}^{\prime} z_{2}^{\prime} \cdots z_{m}^{\prime}\right)^{3 / 2}}=\frac{1}{\Delta^{3 / 2}},
$$

and thus, as with (16), the $z_{i j}$ in (61) do not need to be individually known; only the product $\Delta$, which is invariant under this orthogonal transformation, is required. Therefore the transformation that diagonalizes (59) never has to be explicitly found. The final result is

$$
\begin{aligned}
& N_{m L}\left(\mathbf{p}_{i}, s_{i}\right)=\frac{2^{3 m} \pi^{3(m+L) / 2} \Gamma\left(n+\sum_{i} m_{i}-3 L / 2+3 m / 2\right)}{\left(m_{1}\right) !\left(m_{2}\right) ! \cdots\left(m_{n}\right) !} \\
& \times \int_{0}^{1} d \alpha_{1} \int_{0}^{1} d \alpha_{2} \cdots \int_{0}^{1} d \alpha_{n} \alpha_{1}^{m_{1}} \alpha_{2}^{m_{2}} \cdots \alpha_{n}^{m_{n}} \delta\left[1-\sum_{i=1}^{n} \alpha_{i}\right) \frac{\Lambda^{3(m-1) / 2+n+\sum_{i} m_{i}-3 L / 2}}{\Delta^{3 / 2}(C \Lambda-E+\zeta / \Delta)}{ }^{n+\sum_{i} m_{i}-3 L / 2+3 m / 2} .
\end{aligned}
$$

\section{CONCLUSION}

This systematic method for calculating the Fourier transform of products of standard functions in atomic and molecular physics, and the reduced form of subsequent integrals, removes the time consumption and possibilities for error inherent in an individualized approach.
The reduction in the dimensionality of the integrals of individual problems differing only in the number of products, the indices $\lambda$ and $\eta$, and in the relations between external momenta, must follow the same general path. In the present paper a method has been found that makes this generality explicit. The final form of the Fourier transform of the general product is given and the calculus 
for finding the reduced form of subsequent integrals is done; one is left with just the algebraic operations of constructing (13) and then calculating its determinant (20) and the determinant (21). With the present result given once and for all, more complex theories and systems may be easily studied and more time can be devoted to understanding the physics of these systems.

\section{ACKNOWLEDGMENTS}

This work was done while the author was a guest scientist conducting research through the National Research Council-NASA Research Associateship Program. Computer costs were funded by NASA-RTOP Grant No. 681-170-10-01-08.
*Present address: Department of Physics, Kansas State University, Manhattan, KS 66506.

${ }^{1}$ R. P. Feynman, Phys. Rev. 76, 769 (1949).

${ }^{2}$ B. Podolsky and L. Pauling, Phys. Rev. 34, 109 (1929). This derivation is missing a $(-i)^{l}$ phase factor that is given correctly in E. J. Weniger, J. Math. Phys. 26, 276 (1985), Eq. (4.30).

${ }^{3}$ J. C. Straton, Phys. Rev. A 35, 2729 (1987).

${ }^{4}$ J. C. Straton, Phys. Rev. A 35, 3725 (1987); M. D. Girardeau, ibid. 26, 217 (1982), and references therein.

${ }^{5}$ D. M. Fromm and R. N. Hill, Phys. Rev. A 36, 1013 (1987).

${ }^{6}$ H. P. Trivedi and E. O. Steinborn, Phys. Rev. A 27, 670 (1983).

${ }^{7}$ B. R. Junker, J. Phys. B 13, 1049 (1980); C. Guidotti, G. P. Arrighini, and F. Marinelli, Theor. Chim. Acta 53, 165 (1979); J. Avery and P. Ormen, Int. J. Quantum Chem. 18, 953 (1980).

${ }^{8}$ J. Grotendorst and E. O. Steinborn, J. Comput. Phys. 61, 195 (1985); A. W. Niukkanen, J. Math. Phys. 25, 698 (1984).

${ }^{9}$ E. J. Weniger, J. Math. Phys. 26, 276 (1985).

${ }^{10}$ A. K. Bhattacharya and S. C. Dhabal, J. Chem. Phys. 84, 1598 (1986); E. J. Weniger and E. O. Steinborn, ibid. 87, 3709 (1987).

${ }^{11}$ J. S. Alper, J. Chem. Phys. 55, 3780 (1971), Eq. 18 with $R_{2}=0$. The assertion in this paper that the series was finite was corrected by K. G. Kay and J. S. Alper, ibid. 56, 4243 (1972).

${ }^{12}$ E. J. Weniger and E. O. Steinborn, J. Chem. Phys. 78, 6121 (1983).

${ }^{13}$ E. Filter and E. O. Steinborn, Phys. Rev. A 18, 1 (1978), Eq. (2.14).

${ }^{14}$ A. W. Niukkanen, Int. J. Quantum Chem. 25, 941 (1984); 25, 957 (1984).

${ }^{15}$ Given in H. P. Trivedi and E. O. Steinborn, Phys. Rev. A 27,
670 (1983) with the coefficient corrected to read $(2 / \pi)^{1 / 2}$ in E. J. Weniger, J. Math. Phys. 26, 276 (1985).

${ }^{16}$ Review articles on such problems include F. R. Burden and R. M. Wilson, Adv. Phys. 21, 825 (1972); B. H. Bransden, Adv. At. Mol. Phys. 1, 85 (1965); B. H. Bransden and R. K. Janev, ibid. 19, 1 (1983); and J. T. Park, ibid. 19, 67 (1983).

${ }^{17}$ J. S. R. Chisholm, Proc. Cambridge Phil. Soc. 48, 300 (1952).

${ }^{18}$ H. B. Dwight, Tables of Integrals and other Mathematical Data (Macmillan, New York, 1961), p. 225, No. 859.004. For the case $\eta=0$ this transform is defined only in the sense of a distribution.

${ }^{19}$ S. S. Schweber, An Introduction to Quantum Field Theory (Harper and Row, New York, 1962), p. 913.

${ }^{20}$ The explicit form of $(8)$ in this case is given in C. J. Joachain, Quantum Collision Theory (North-Holland, Amsterdam, 1983), p. 678.

${ }^{21}$ I. S. Gradshteyn and I. M. Ryzhik, Table of Integrals, Series, and Products (Academic, New York, 1980), p. 317, No. 3.381(4)

${ }^{22}$ Ref. 21 , p. 340 , No. $3.471(9)$.

${ }^{23}$ Ref. 21 , p. 413 , No. $3.737(2)$.

${ }^{24}$ I. M. Cheshire, Proc. Phys. Soc. 83, 227 (1964).

${ }^{25}$ See, for instance, Eq. (B9) in J. C. Straton, Phys. Rev. A 35, 3725 (1987).

${ }^{26}$ One could instead define, for any value of $\rho, \mathbf{y}_{j}=i \mathbf{x}_{j} / 2 \rho$. Then $H=-Y^{T} Z Y$, where $Y^{T}=\left[\mathbf{y}_{1}, \mathbf{y}_{2}, \ldots, \mathbf{y}_{m},=1\right]$, and $H^{\prime}=-z_{1}^{\prime} y_{1}^{\prime 2}-z_{2}^{\prime} y_{2}^{\prime 2}-\cdots-z_{m}^{\prime} y_{m}^{\prime 2}+g^{\prime}$, leading to (64). Finally one substitutes $\mathbf{y}_{j}^{\prime}=i \mathbf{x}_{j}^{\prime} / 2 \rho$ to obtain (62).

${ }^{27}$ Ref. 21, p. 705, No. 6.596(3).

${ }^{28}$ Ref. 21 , p. 684 , No. $6.561(17)$. 